\title{
Représenter la victoire militaire d'une femme
}

le cas de l'étendard de la reine Gerberge

Representation of the military victory of a woman: the standard of Queen

Gerberge

\section{Marjolaine Massé}

\section{OpenEdition}

\section{Journals}

Édition électronique

URL : http://journals.openedition.org/cel/9017

DOI : $10.4000 /$ cel.9017

ISSN : 2262-208X

Éditeur

École du Louvre

Référence électronique

Marjolaine Massé, «Représenter la victoire militaire d'une femme », Les Cahiers de l'École du Louvre [En ligne], 15 | 2020, mis en ligne le 02 novembre 2020, consulté le 05 novembre 2020. URL : http:// journals.openedition.org/cel/9017 ; DOI : https://doi.org/10.4000/cel.9017

Ce document a été généré automatiquement le 5 novembre 2020.

\section{()) $(9$}

Les Cahiers de l'École du Louvre sont mis à disposition selon les termes de la licence Creative Commons Attribution - Pas d'Utilisation Commerciale - Pas de Modification 4.0 International. 


\title{
Représenter la victoire militaire d'une femme
}

\author{
le cas de l'étendard de la reine Gerberge \\ Representation of the military victory of a woman: the standard of Queen \\ Gerberge
}

Marjolaine Massé

1 La représentation du pouvoir des femmes dans les images du haut Moyen Âge, et plus particulièrement au début de la dynastie ottonienne, est un véritable enjeu car c'est à cette époque que l'on assiste progressivement à l'apparition puis au développement des images de femmes de pouvoir. Même s'il existe quelques études consacrées aux images des femmes du haut Moyen Âge, rares sont les historiens et les historiennes de l'art qui font du genre leur axe principal de recherche ${ }^{1}$. Or, si dès l'époque carolingienne, certaines femmes de l'aristocratie jouent un rôle politique crucial et commanditent des objets précieux, elles sont très peu représentées dans les images. Les images de femmes sont en effet symptomatiques de leur place au sein d'un échiquier politique brutal et largement masculin. Dans ce contexte, et au prisme des gender studies, la bannière de Gerberge, unicum à l'aune du XXI siècle, permet de questionner le rôle du genre dans l'iconographie des femmes de l'élite impériale ottonienne.

2 La bannière de Gerberge est une broderie de forme carrée mesurant $37 \mathrm{~cm}$ de côté, conservée au trésor de la cathédrale de Cologne ${ }^{2}$. Elle porte l'inscription « Gerberga me fecit », "Gerberge m'a fait », qui identifie la reine Gerberge comme commanditaire de l'œuvre ${ }^{3}$ (fig. 1).

3 Le terme "violence " apparaît en français dans le premier quart du XIII ${ }^{\mathrm{e}}$ siècle, soit près de deux siècles et demi après la réalisation de la bannière de Gerberge. Étymologiquement, le terme vient du latin violentia, qui dérive du mot vis, qui signifie force, puissance. Or, c'est véritablement une démonstration de puissance qui se joue avec cette image et passe par, à la fois, la matérialité de l'objet et son iconographie triomphale, symptomatiques de la violence qui s'exerce à l'époque dans les rapports de force pour le pouvoir au sein de l'entourage impérial. 
4 Après une présentation du contexte de production de l'objet lui-même, ainsi que de sa commanditaire, la reine Gerberge, nous analyserons comment l'iconographie et les inscriptions qui se trouvent sur cette bannière en font un outil de démonstration du pouvoir de la reine. Dans cette affirmation de puissance, fruit d'un contexte historique spécifique, la bannière reflète la relation particulière des reines, à la fois au pouvoir et à l'art de la broderie. Le medium de la broderie est en effet un outil d'expression capital pour les femmes de l'élite durant le haut Moyen Âge.

Fig. 1

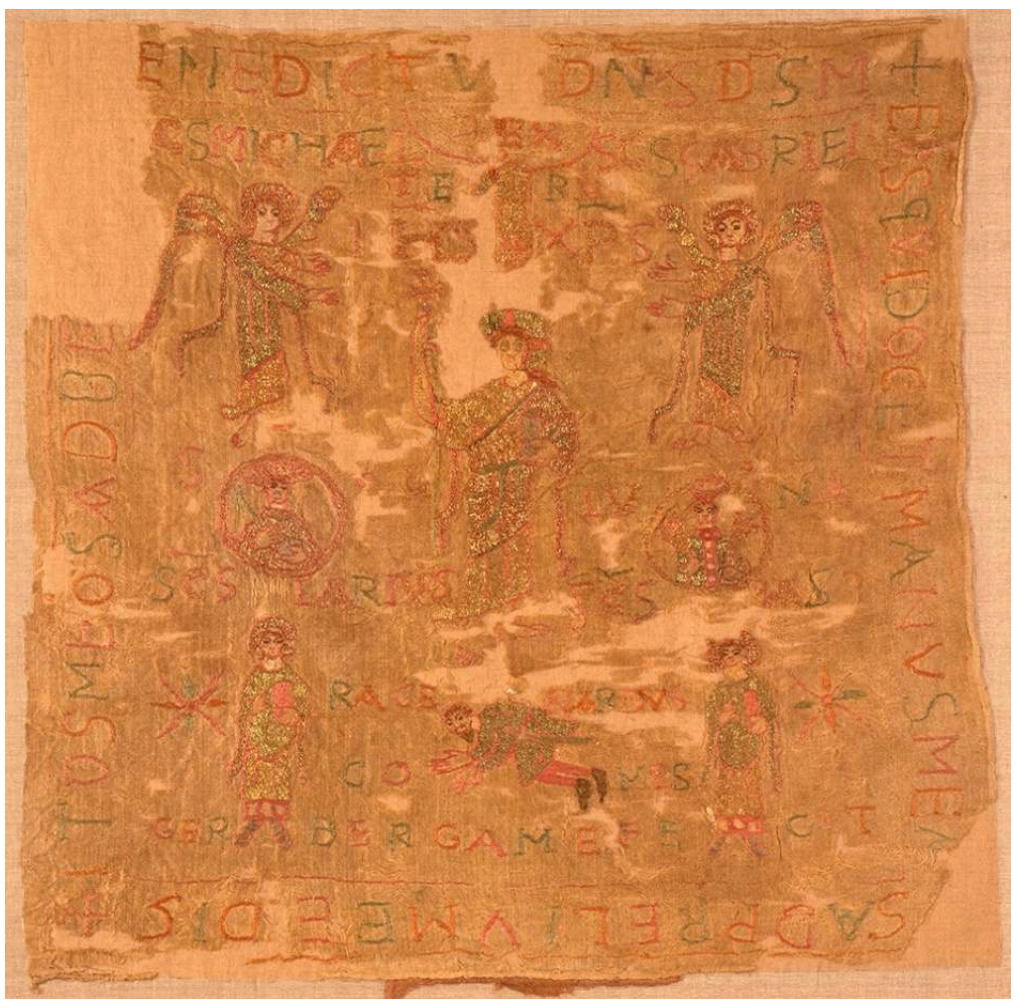

Bannière de Gerberge, vue d'ensemble, vers 957, Trésor de la Cathédrale, Cologne. (c) Hohe Domkirche Köln, Dombauhütte Köln, Foto : Matz und Schenk

\section{Le contexte d'une production royale}

La bannière de Gerberge, réalisée à la demande de la reine, est extrêmement précieuse et d'ampleur royale, de par le coût, la rareté et la préciosité de ses matériaux. Ce textile commémoratif en soie brodé d'or a été tissé vers 957, probablement à Laon. Le fond est une étoffe mi-lin mi-soie, autrefois de couleur rouge-orangée, aujourd'hui passée. Il est brodé de fils de soie rouges, verts, bruns et de fils d'or. L'image reste très lisible malgré des lacunes situées principalement au niveau du fond, et non au niveau des parties brodées. L'étoffe qui constitue le fond est construite selon une trame assez aérée permettant la broderie avec des fils épais car recouvert de métal, le fil d'or étant obtenu en enroulant une fine lamelle d'or autour d'un fil. C'est pourquoi le fond, plus fragile, est davantage abîmé que les broderies elles-mêmes.

Cette œuvre s'inscrit dans une tradition anglo-saxonne de la broderie, mais témoigne aussi de la prégnance des modèles byzantins, tant par les modèles véhiculés que par les 
matières employées. Les matériaux ayant servi à la réalisation de la bannière de Gerberge ont en effet été importés, la soie n'étant pas filée en Europe à cette époque. Elle est réservée à l'aristocratie et on la retrouve par exemple en contexte funéraire dans les tombes de rois, de reines ou de saints. La plupart des textiles en soie qui se trouvaient en Europe venaient de Byzance. Les médiévaux importaient à la fois des textiles proprement byzantins, ornés d'une iconographie byzantine et des matériaux destinés à être employés pour réaliser des œuvres textiles locales, comme c'est le cas ici. Les œuvres et l'iconographie byzantines sont de plus en plus présentes en Occident au Xe siècle, notamment à la cour ottonienne ${ }^{4}$.

7 La commanditaire, Gerberge, possède une identité politique triple en tant que reine, sœur de l'empereur et veuve du duc. Ce sont ces circonstances exceptionnelles, notamment sa fratrie et ses veuvages, qui en font une figure remarquable. Son statut et ses prérogatives de reine la désignent comme la commanditaire logique de cet objet, dont l'iconographie traduit le caractère politique et impérial.

Gerberge (913-969/984) est une aristocrate membre de la famille impériale ottonienne. Ses frères sont Otton, empereur d'Occident, et Brunon, archevêque de Cologne. Sa sœur Hedwige est l'épouse de Hugues le Grand et donnera naissance à Hugues Capet vers 939-941. Gerberge devient d'abord duchesse de Lotharingie en épousant Gislebert de Lotharingie en 928. Celui-ci meurt rapidement et elle se remarie avec le roi de Francie occidentale Louis IV d'Outremer. Personnage véritablement au cœur de l'échiquier politique ottonien, elle est duchesse de Lotharingie entre 928 et 939, puis reine de Francie occidentale entre 939 et 954 . Femme extrêmement active dans la vie politique et religieuse de son temps, elle mène, en 957 , une campagne militaire avec l'aide de sa famille, contre son neveu le comte Régnier de Hainaut, qui menaçait de l'envahir. Elle triomphe ${ }^{5}$ et c'est à cette occasion qu'elle aurait fait réaliser cette bannière, représentant le Christ victorieux, aux pieds duquel Régnier, comte de Liège et de Hainaut, apparaît prosterné, identifié par l'inscription « Ragenardus comes ».

Elle est donc liée à deux dynasties : celle des carolingiens (par son second mariage) et celle des ottoniens (par le sang) et joue un rôle de médiatrice entre son mari et ses frères de par sa position entre les dynasties d'est et d'ouest. Le pouvoir de Gerberge ne se limite pas à sa naissance et à ses mariages. Elle est aussi active dans les luttes de pouvoir. Par exemple, lorsque son premier mari est fait prisonnier par les Normands, puis par Hugues le Grand en 945-946, c'est elle qui négocie sa libération et qui demande de l'aide à son frère Otton. Elle agit également seule en 949 , en se chargeant d'une mission diplomatique et se rend à Aix-la-Chapelle auprès d'Otton pour lui demander de l'aide ${ }^{6}$. Après la mort de Louis IV en 954, elle assure une forme de régence jusqu'en 962 environ, puis son fils Lothaire se défait progressivement de son autorité. Gerberge opère aussi dans ces années-là un rapprochement diplomatique avec son frère Brunon, archevêque de Cologne.

10 Les activités monastiques constituent une part importante du pouvoir des reines et c'est probablement dans ce contexte que fut réalisée la bannière de Gerberge. Il existe depuis l'époque mérovingienne une tradition de monastères dirigés par des souveraines, dans laquelle Gerberge s'inscrit. Dans le contexte monastique, les nonnes produisent les textiles commandités par les reines, malgré leur accès a priori limité au monde et au pouvoir. Le contrôle des monastères est un aspect important du Queenship ${ }^{7}$, qui s'amplifie à l'époque carolingienne. C'est une activité essentielle qui constitue une des bases du pouvoir carolingien et pas seulement du pouvoir des 
femmes. Le pouvoir exercé par les reines par l'intermédiaire des monastères leur permet d'asseoir leur autorité et ce même après la mort du roi. Ces responsabilités monastiques sont l'occasion pour les femmes d'exercer leur pouvoir en dehors des limites posées par leur statut au sein de la famille, et de mener à bien des chantiers artistiques, de commanditer et de concevoir des œuvres.

Gerberge est une grande mécène, conformément à ses prérogatives de reine, dont le mécénat ne se limite pas aux objets précieux, mais s'étend aussi aux lettres, comme l'a montré l'historien Simon MacLean ${ }^{8}$. Elle commande par exemple à l'abbé Adso, un traité sur l'origine de l'Antichrist, composée entre 949 et 954. Cet intérêt de la reine pour les questions théologiques et pour la commande artistique doit être compris au regard de son activité politique laïque et ecclésiastique. Les objets et les textes qu'elle commandite, ainsi que son intérêt pour l'antichrist et pour la fin du monde, sont le reflet de son statut dynastique, de ses intérêts de reine pour les affaires monastiques et de son rôle à la cour. Dans la dédicace de son traité sur l'Antichrist, Adso s'adresse à Gerberge comme à une connaisseuse des questions théologiques et monastiques ${ }^{9}$, car les reines sont, depuis l'époque carolingienne, très au fait des questions d'exégèse et sont en mesure d'exprimer un avis éclairé sur le sujet. Par son mécénat et son rôle d'abbesse, Gerberge s'impose comme une véritable leader monastique au sein de l'élite impériale et pas seulement en tant que reine, épouse, fille, sœur, mère.

12 Les femmes peuvent, au $\mathrm{X}^{\mathrm{e}}$ siècle, exercer l'autorité royale, car les barrières qui les tiennent à l'écart du pouvoir ne sont pas institutionnelles ${ }^{10}$. L'appartenance à l'élite est, en revanche, un facteur décisif : le rang social est pour Gerberge plus déterminant que son genre. En effet, au haut Moyen Âge, les individus sont davantage déterminés par leur classe sociale que par leur genre. Néanmoins, la société du $\mathrm{X}^{\mathrm{e}}$ siècle reste tout de même très patriarcale et les reines ne peuvent exercer leur autorité que si elles sont rattachées à un homme, mari ou fils, en général. Il règne également une hiérarchie au sein du couple, soumettant la femme à l'homme. C'est pourquoi le pouvoir d'une reine telle que Gerberge se développe particulièrement au moment de son veuvage ${ }^{11}$.

L'importance du rôle militaire de Gerberge est relativement rare chez une reine, mais il n'est pas exceptionnel. Il existe quelques autres exemples, comme celui de la reine Emma, qui s'était aussi battue aux côtés de son mari Raoul, le roi des Francs, et avait organisé la défense de la ville de Laon en 927, ou encore Aethelflaed, reine de Mercie entre 911 et 918, qui remporte également des victoires militaires. Mais Gerberge et Aethelflaed ont pour point commun d'être veuves, ce qui facilite leur (relative) émancipation ${ }^{12}$. La femme cheffe de guerre reste rare et concerne une minorité de personnalités féminines atypiques.

\section{Iconographie impériale et ordonnancement du monde}

14 Au centre de la bannière de Gerberge se trouve le Christ victorieux, reconnaissable à son nimbe crucifère, tenant une croix et un livre, et surmonté de l'inscription IHS/XPS, son monogramme. Au-dessus de lui se trouve la main de Dieu accompagnée de l'inscription "dextera». Il est entouré des archanges Michel et Gabriel et des personnifications du soleil et de la lune, Sol et Luna, héritées de l'imagerie impériale romaine, mais qui sont aussi des éléments de l'iconographie de la crucifixion. rendant hommage au Christ, est issue d'une longue tradition qui se perpétue dans 
l'Empire ottonien. Elle remonte aux représentations d'empereurs romains triomphant encadrés par deux soldats et deux victoires, dont l'ivoire Barberini conservé au musée $\mathrm{du}$ Louvre est sans doute l'exemple le plus célèbre. Notre image peut aussi être rapprochée, par sa composition, d'autres d'images impériales ottoniennes, comme le folio $11 \mathrm{du}$ sacramentaire de l'empereur Henri II ${ }^{13}$ (fig. 2). On y voit l'empereur couronné par le Christ, soutenu par deux saints et entouré de deux victoires. Ce schéma iconographique vise à célébrer la victoire et la grandeur du Christ ou de l'empereur.

Fig. 2

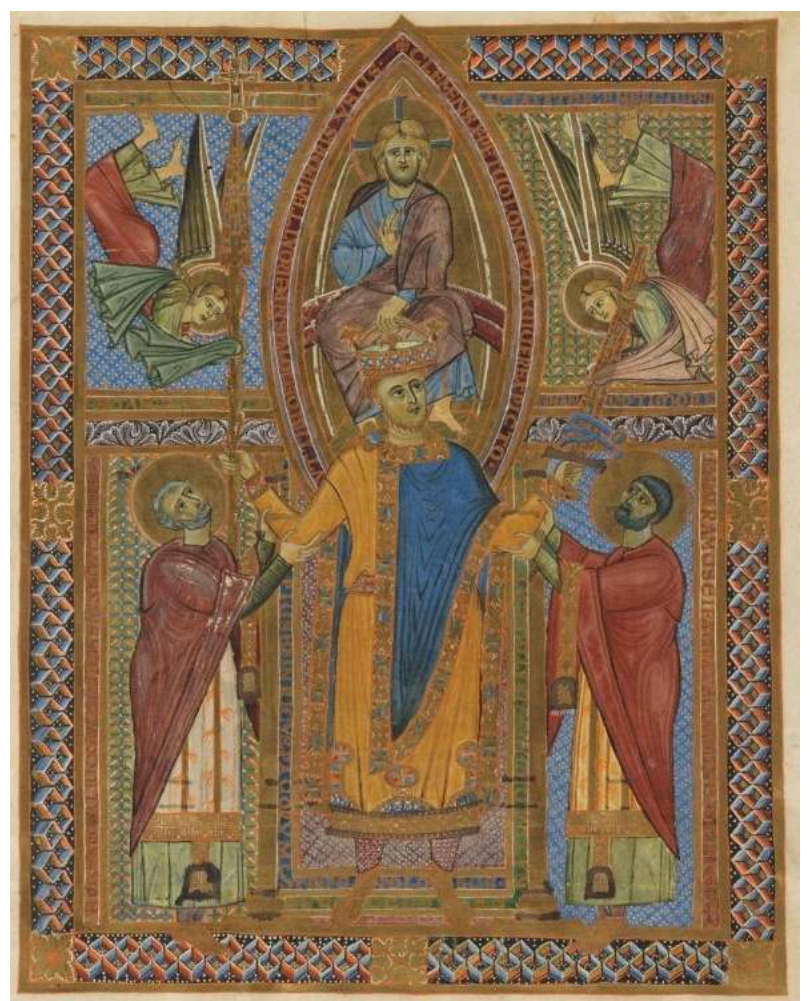

Enlumineur du scriptorium de Ratisbonne, «Le couronnement d'Henri II », Sacramentaire d'Henri II, 1002-1014

(c) Bayerische Staatsbibliothek

Sur la bannière de Gerberge, on trouve aussi le comte Régnier de Hainaut sous les pieds Christ, en position de proskynèse. Il est entouré des saints Bason et Hilaire, tous deux martyrisés à Laon. Il s'agit d'une combinaison de saints inhabituelle qui rattache cet objet à la ville de Laon et plaide en faveur d'une réalisation locale. Laon est un des pôles de la faible autorité de Louis IV, et Gerberge entretient un lien très fort avec la ville qu'elle reprend militairement à Hugues le Grand en $949^{14}$. À partir de 951, Laon est une résidence de Gerberge, où elle prend la direction de l'abbaye bénédictine de SainteMarie $^{15}$.

L'autorité de Dieu domine au centre de l'image à travers le Christ. Les figures des archanges et des saints, du soleil et de la lune sont disposés autour de lui, produisant une composition symétrique et stable que vient troubler de Hainaut (fig. 3). Il est l'élément qui permet de rompre la frontalité et la symétrie de l'image. 
Fig. 3

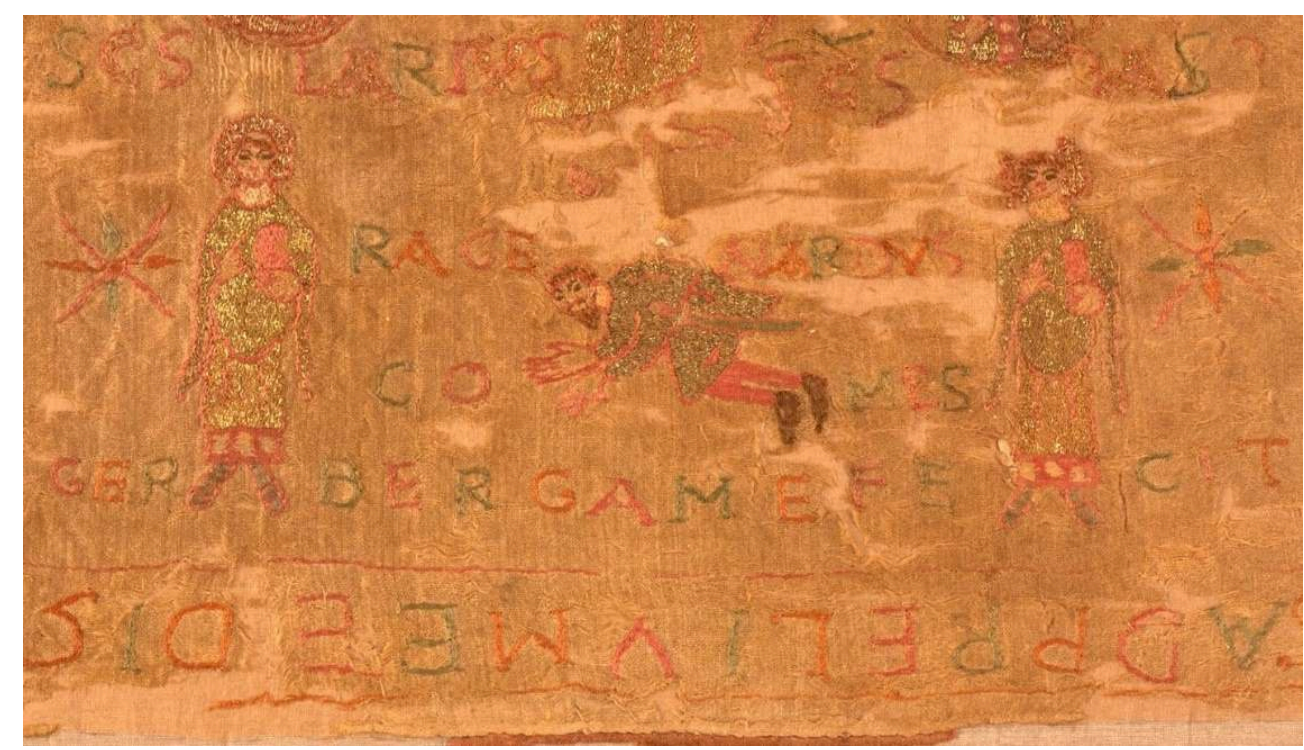

Bannière de Gerberge, vue de détail, vers 957, Trésor de la Cathédrale, Cologne

(c) Hohe Domkirche Köln, Dombauhütte Köln, Foto: Matz und Schenk

L'appartenance de Régnier au simple monde terrestre est signifiée par l'absence de nimbe et par sa tenue. Il porte un vêtement militaire composé d'une tunique courte, des braies rouges portées par l'élite franque que l'on retrouve sur d'autres images de l'époque et porte son épée au fourreau. Sa tenue le caractérise comme faisant partie de l'élite militaire franque. La différence d'échelle entre les personnages exprime aussi une hiérarchie, qui met Régnier à sa place, c'est-à-dire en position d'humilité, de petite taille sous les pieds du Christ. Même s'il serait tentant d'adapter l'iconographie aux circonstances historiques, mieux vaut rester sur le matériau à disposition. Cette posture d'humilité est courante dans les images de donateurs et de commanditaires de l'époque. On la retrouve par exemple chez Arnulf de Carinthie au pied de la croix dans le psautier de Louis le Germanique ${ }^{16}$ (fig. 4), ou encore chez Thierry de Frise et Hildegarde de Flandres dans l'évangéliaire d'Egmond ${ }^{17}$. Il n'est donc pas représenté dans une position de soumission particulière. L'hypertrophie des mains se retrouve également dans de nombreuses images carolingiennes et ottoniennes et sert à accentuer la gestuelle dans le cadre d'images hautement symboliques. Ici, elle sert à appuyer la gestuelle de l'orant. 
Fig. 4

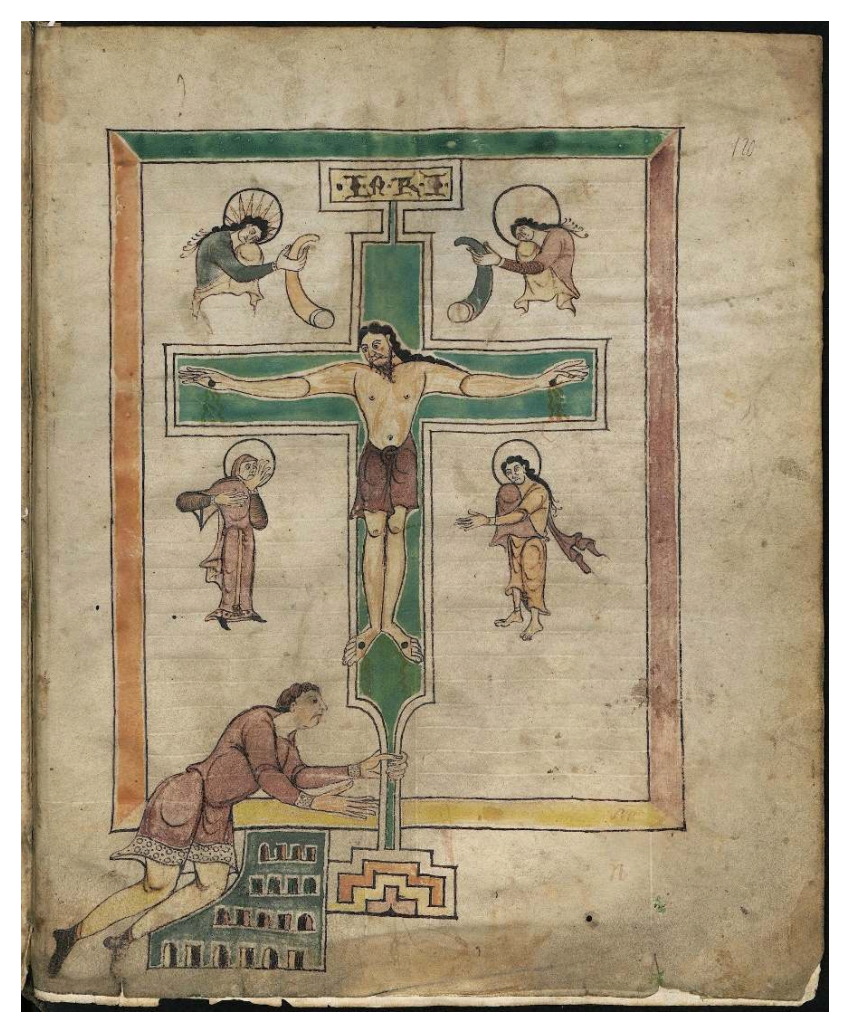

Anonyme, "Crucifixion avec Arnulf de Carinthie au pied de la croix », Psautier de Louis le Germanique, vers 850 .

(c) Staatsbibliothek zu Berlin - Preußischer Kulturbesitz

Tout autour de l'image court une inscription tirée du $1^{\mathrm{er}}$ verset du Psaume 143 : «Benedictus Dominus Deus meus, qui docet manus meas ad praelium, et digitos meos ad bellum " soit : "Béni soit le Seigneur, mon rocher, qui entraîne mes mains pour le combat, mes poings pour la bataille ${ }^{18}$.». De par sa disposition, l'inscription encadre l'image et la conditionne dans une signification guerrière, renforcée par la présence des archanges et du Christ victorieux. Ce dispositif et les différents éléments de l'image créent un complexe visuel, qui rend compte d'un ordre du monde. L'image n'est pas ancrée dans l'histoire, elle ne possède pas de temporalité et se situe hors du temps, dans un espace symbolique. Contrairement à d'autres images, comme celle du psautier de Louis le Germanique, il n'y a pas ici de contact entre les personnages : or, le contact manifeste la transmission. Sur cette image, il n'y a pas de transmission de l'autorité, chacun reste à sa place, conformément à la volonté carolingienne d'ordre social.

Il s'agit d'un objet principalement séculier malgré son iconographie éminemment chrétienne. Il commémorerait la défaite et l'exil du comte Régnier, vers 958-960. La situation politique est, en effet, plutôt tendue dans les années 950. Cette époque voit le délitement de l'Empire carolingien et l'avènement de celui des ottoniens. La situation est globalement instable et génère beaucoup de tensions et conflits, principalement pour des questions territoriales. Régnier, neveu du premier mari de Gerberge, profite $\mathrm{du}$ désordre pour se rebeller contre Otton, Brunon et Gerberge, et envahit des territoires appartenant à celle-ci en Lotharingie. En représailles, elle envoie des espions puis des hommes d'armes, détruire le château de Régnier et fait enlever sa femme et ses 
enfants. Mais cela ne suffit pas, Régnier continuera d'envahir la Lotharingie, jusqu'à ce que Brunon finisse par l'exiler en $957^{19}$.

21 Mais plus que la commémoration d'un événement particulier, cet objet rend compte d'une situation politique et affirme la place de chacun. Cela s'explique par le contexte d'insécurité dans lequel il est produit: rivalité pour le trône de France et plus largement rivalité pour le pouvoir, qui n'est plus centralisé par l'empereur, mais par quelques grandes familles. Des tensions ont lieu entre ces familles, voire en leur sein même, car dans l'entourage de Gerberge, les membres de la famille impériale se battent entre eux pour le pouvoir. Le rôle des femmes en politique est à cette époque primordial. Elles sont souvent les plus fidèles alliées de leurs maris car ayant moins accès au pouvoir, elles sont plus dépendantes et leur place dans la société, voire leur survie, en dépend. Un objet comme la broderie de Gerberge est symptomatique de leur pouvoir informel. Il faut noter que ces femmes cheffes de guerre du haut Moyen Âge agissent le plus souvent pour le bien de leur entourage, mais parfois aussi pour leurs propres fins. Dans le cas de Gerberge, les deux vont de pair : elle mène sa vie politique de manière à stabiliser sa lignée et sa propre position, les deux étant interdépendants.

La puissance de Gerberge se manifeste de manière visible par l'inscription qui se trouve sur la broderie. Celle-ci est en effet symptomatique des liens étroits entre le texte et l'image dans l'art médiéval, car les deux sont pensés ensemble et participent à l'unité visuelle de l'objet. Ici, ce sont les inscriptions qui, en relation étroite avec l'image, portent le message de l'œuvre. C'est pourquoi la reine est seulement présente par l'écriture, qui suffit à la représenter car la dédicace "Gerberga me fecit » est porteuse de suffisamment d'autorité. Elizabeth Coatsworth ${ }^{20}$ avait déjà remarqué que la plupart des broderies occidentales médiévales conservées portent des inscriptions, qui peuvent avoir quatre fonctions. La première est de commémorer le souvenir du donateur, du fabriquant ou du destinataire de l'œuvre. La deuxième est d'identifier quelqu'un ou quelque chose sur l'image, la troisième est la fonction narrative et la quatrième est d'avoir une valeur en soi, presque indépendamment du reste de l'image et conduire le regardant vers une signification plus profonde de l'objet ou de l'iconographie. Les inscriptions de la bannière de Gerberge rentrent dans plusieurs de ces fonctions. Certaines servent à identifier les personnages, mais l'objet, dans son ensemble, commémore une situation historique. L'inscription «Gerberga me fecit » identifie la commanditaire et « Ragenardus comes », le comte Régnier. L'inscription qui fait le tour de l'image, celle du verset 1 du Psaume 143, concorde avec la thématique guerrière, car il s'agit d'une prière en remerciement après une victoire militaire. Il a pour sujet précis le combat de David et Goliath, mais évoque plus largement tout type de combat et permet d'inscrire l'image dans un contexte guerrier.

Ces éléments textuels et iconographiques nous donnent un certain nombre d'indices sur les raisons de la commande d'un tel objet: Gerberge donne à voir la mise en ordre hiérarchisée du monde, tout en mettant en avant son rôle en tant que commanditaire d'un objet extrêmement précieux, conformément à ses prérogatives de reine. Elle affirme ainsi sa place dans l'échiquier politique.

\section{L'emploi d'un medium genré}

L'utilisation à ces fins de la broderie n'est pas anodine car il s'agit d'un medium éminemment genré. L'étendard de Gerberge s'inscrit en effet dans une longue tradition 
associant les femmes de l'élite et particulièrement les reines, à la pratique de la broderie et au don de textiles précieux. La commande de manuscrits et la production textile étaient les sphères dans lesquelles la contribution des élites féminines avait le plus de valeur au Moyen Âge ${ }^{21}$. À cette époque, les broderies étaient souvent des objets exceptionnels et demandaient énormément de temps et des matériaux coûteux, reflet du haut rang de leur commanditaire. Peu de broderies médiévales nous sont parvenues, car ce medium possède un caractère bien plus périssable que les métaux précieux, les ivoires, ou même le parchemin. Il est donc difficile de mesurer l'importance de cette production au sein de l'ensemble de la production artistique de l'époque. De plus, lorsque des textiles sont conservés, ils le sont grâce à leur rapport au sacré. C'est le cas de la bannière de Gerberge, utilisée pour envelopper des reliques. ${ }^{22}$ La reine l'a d'abord offerte à son frère Brunon, archevêque de Cologne, qui l'a ensuite utilisée pour envelopper les reliques de Grégoire de Spolète dans la châsse des Rois mages de la cathédrale de Cologne.

La broderie est un medium traditionnellement associé aux femmes, mais il est difficile d'estimer la part de stéréotype. Il est souvent impossible de déterminer avec certitude le sexe des artisans. Les travaux d'aiguille sont considérés comme un passe-temps acceptable pour les femmes de l'aristocratie, selon une tradition qui remonte à l'Antiquité et qui se justifie dans le contexte chrétien du Moyen Âge par la volonté de se référer à la Vierge filant la pourpre ${ }^{23}$. Les hommes brodent aussi : les moines peuvent par exemple le faire dans le cadre de leurs travaux manuels dans le monastère. Même si l'on sait que les femmes brodaient, les conditions de production précises des œuvres, à la cour ou dans les couvents, restent souvent inconnues. On peut néanmoins fortement supposer, d'après le contexte, que la plupart des broderies du haut Moyen Âge qui nous sont parvenue ont été réalisées dans des monastères et dans le cas de la bannière, probablement dans celui de Sainte-Marie de Laon dirigé par Gerberge. Cette localisation est importante d'un point de vue symbolique (car elle permet d'ancrer l'objet dans un territoire royal carolingien) et militaire (car il s'agit d'un territoire au centre du pouvoir et d'une zone pivot, importante stratégiquement). Ici, la commande et la conception de l'image ont probablement été ordonnées par Gerberge et la réalisation de la broderie confiée aux nonnes. Car cet art requiert un très grand degré d'expertise, de savoir-faire, mais surtout beaucoup de temps, ce dont Gerberge ne disposait pas car elle participait à de nombreuses intrigues politiques et voyageait beaucoup pour stabiliser sa position et celle des siens dans l'Empire ${ }^{24}$.

Les femmes sont peu visibles dans les œuvres du haut Moyen Âge, à la fois dans l'iconographie et dans la commande et la conception des œuvres. La principale raison à cela est le poids de la société patriarcale chrétienne et guerrière du $\mathrm{X}^{\mathrm{e}}$ siècle. Elles ont souvent des rôles d'intercessrices, de médiatrices ou de conseillères qui leur confèrent un " pouvoir informel » qui est moins perceptible et moins reconnu, car il ne s'exerce pas au nom d'un "titre» institutionnel. Ce pouvoir informel contribue à leur invisibilisation. C'est probablement pour cela que l'historiographie sur le pouvoir a longtemps minoré la place des femmes.

À ce manque de visibilité s'ajoute l'ambiguïté des inscriptions de dédicace au Moyen Âge : la personne qui fait, qui conçoit, qui entreprend ou qui tient le rôle de mécène peut être désignée comme celle qui «fait» par l'expression "me fecit». Quand une inscription dit qu'une femme de l'élite a «fait » une œuvre, cela veut aussi bien dire qu'elle a pu la commanditer, fournir les matériaux précieux, superviser sa réalisation, 
ou bien la réaliser elle-même. Selon Annmarie Weyl Carr ${ }^{25}$, certaines attributions sont dues au fait que les femmes sont traditionnellement associées aux travaux d'aiguille. Elle prend pour cela l'exemple du «manteau de Cunégonde», pluvial liturgique du début $\mathrm{du} \mathrm{XI}^{\mathrm{e}}$ siècle ${ }^{26}$ dont la réalisation a longtemps été attribuée à l'impératrice Cunégonde, épouse d'Henri II. Selon l'autrice, si l'inscription avait désigné un personnage masculin, il aurait été plus volontiers identifié comme le commanditaire, et non le faiseur de l'œuvre. Cunégonde est désormais regardée comme la commanditaire, mais cet exemple témoigne d'une tendance aujourd'hui dépassée de l'historiographie à attribuer la réalisation des textiles systématiquement, ou presque, aux femmes; la commande et la conception étant plus facilement attribuées aux hommes.

La commande de textiles précieux par l'élite féminine est également attestée par des témoignages textuels. On sait par exemple qu'Aefflaed, épouse du comte d'Essex Byrhnoth, fait réaliser à la fin du $\mathrm{X}^{\mathrm{e}}$ siècle une broderie racontant la bataille de son mari contre les vikings ${ }^{27}$, qu'elle offre à la cathédrale d'Ely. Une autre Aefflaed, épouse d'Édouard l'Ancien, est aussi à l'origine de la commande des vêtements liturgiques de saint Cuthbert, une manipule et une stola, conservées au trésor de la cathédrale de Durham. Les inscriptions placées au revers des extrémités de la stola «Aelfflaed fieri precepit " et «Pio episcopo Fridestano» précisent bien que la reine en était la commanditaire et non l'exécutante. Il existe une tradition, plus développée dans le monde anglo-saxon, de commande et de dons de broderies par des aristocrates laïques à des établissements religieux. La bannière de Gerberge s'inscrit dans cette tradition de textiles produits en relation avec des femmes et particulièrement avec des femmes appartenant à l'entourage royal ou impérial. Celle-ci l'offre à son frère, Brunon l'archevêque de Cologne, certainement pour le remercier de son soutien face à ces ennemis.

\section{Usage de l'objet}

Mais le contexte politique ne doit pas effacer la fonction originelle de l'objet, aujourd'hui incertaine. La broderie est un medium utilisé pour démontrer, décorer, ou encore instruire, dans une visée idéologique impériale et/ou chrétienne. L'image est ici hautement symbolique et le texte peut être compris dans une signification à la fois séculière et religieuse. Les dimensions réduites et la préciosité de la bannière ajoutent à la complexité de la lecture. Elle était donc destinée à être vue par une audience réduite, au cour de cérémonies en petit comité, même si en l'absence de contexte précis, il est délicat de déterminer l'usage exact de l'objet. On peut émettre l'hypothèse que tout comme la broderie de Bayeux, il était destiné à être accroché au mur et à commémorer une victoire ${ }^{28}$. Gerberge se rend ainsi visible dans un monde dominé par des signes, des symboles et un cérémonial, souvent contrôlés par les hommes.

Le contexte précis d'utilisation de l'œuvre reste inconnu. Mais sa préciosité lui confère un caractère sacré, qui la fait entrer dans la catégorie des textiles employés dans le cadre des pratiques cultuelles. Les officiants se servent des textiles lors du culte. Ils participent au rite et ils sont également assez précieux et luxueux pour être dignes de toucher les reliques. Le textile est en effet un medium qui a la capacité, de par la préciosité de sa matière, de jouer un rôle de médiateur vers le sacré. Porosité entre le textile et la relique. Les reliques sont en effet fréquemment enveloppées dans des étoffes précieuses, souvent byzantines. La frontière est étroite entre les textiles qui 
sont sacrés par eux-mêmes et les textiles qui le sont parce qu'ils sont au contact de reliques.

\section{Impossibilité de représenter la femme guerrière}

31 L'étendard de Gerberge s'inscrit dans la tradition des œuvres textiles commanditées par les reines et les femmes de l'aristocratie, avec la particularité qu'il s'agit d'un objet vecteur de pouvoir qui rend compte de la position dominante de la reine. Mais l'image se heurte ici à une limite : celle de représenter la femme guerrière. La bannière de Gerberge est un exemple de contournement de cette impossibilité, qui ne pouvait l'être que par le medium de la broderie, allié à la force de l'écrit et à l'iconographie impériale. La production de textiles constitue une exception, une brèche dans laquelle les femmes $\mathrm{du}$ haut Moyen Âge peuvent laisser plus facilement leur trace en tant que commanditaires. Néanmoins, leur violence ne peut pas être représentée dans le cadre de cette société.

Les souverains de cette époque sont en recherche constante de légitimation dynastique vis-à-vis d'un pouvoir qu'ils ont usurpé et cherchent à consolider le pouvoir de leur lignée. La bannière de Gerberge affirme la puissance de la reine, non pas à ses propres fins, mais à celles de sa lignée, en vue d'une recherche de stabilité dans un échiquier politique instable. Cette image met en évidence l'exercice d'une puissance plutôt que d'une violence, car la force qu'elle exerce est légitime.

Cet objet témoigne de la concordance rarissime entre le medium du textile, une situation matrimoniale et familiale exceptionnelle et des conditions qui ont permis sa conservation, à l'intérieur de la châsse des Rois mages de la cathédrale de Cologne.

\section{NOTES}

1. On peut néanmoins citer les articles d'Annmarie Weyl Carr, «Women as artists in the middle ages: the dark is light enough », Dictionary of women artists, London, Royaume-Uni de GrandeBretagne et d'Irlande du Nord, 1997, 2 vol., pp. 3-21 ; Alexandra Gajewski, « Having her hand in it ? Elite women as "makers" of textile art in the Middle Ages ", Journal of Medieval History, vol. 42, 2016, pp. 25-50 ; Valerie L. Garver, «Weaving words in silk: Women and inscribed bands in the Carolingian world. », Medieval clothing and textiles, vol. 6, 2010, pp. 33-56.

2. Elle fût découverte lors de l'ouverture de la châsse en 1864.

3. Anton von Euw, «Zur Ikonographie der sog. Kriegsfahne im Kölner Domschatz », Kölner Domblatt, 21/22, 1963, pp. 37-48.

4. Hiltrud Westermann-Angerhausen, «Did Theophano leave her mark on the Ottonian sumptuary arts?», The Empress Theophano, s. 1., 1995, pp. 244-264.

5. Megan Welton, «Domina et Fidelibus Eius: Elite Households in Tenth-Century Francia and Anglo-Saxon England ", Royal and elite households in medieval and early modern Europe, s. 1., 2018, pp. 15-41.

6. Régine Le Jan, Femmes, pouvoir et société dans le haut Moyen Age, Paris, Picard, 2001, p. 35. 
7. Caroline Walker Bynum, Crown and veil: female monasticism from the fifth to the fifteenth centuries, J. F. Hamburger et S. Marti (éd.), New York, Columbia University Press, 2008 ; Jutta Frings, Kunstund Ausstellungshalle der Bundesrepublik Deutschland, Krone und Schleier. Kunst aus mittelalterlichen Frauenklöstern, München, 2005.

8. Simon MacLean, "Reform, Queenship and the End of the World in Tenth-Century France: Adso's "Letter on the Origin and Time of the Antichrist" Reconsidered ", Revue belge de philologie et d'histoire, vol. 86, $\mathrm{n}^{\circ}$ 3, 2008, pp. 645-675.

9. Idem.

10. Jo Ann Kay McNamara, "Women and power through the family revisited", Gendering the master narrative, s. 1., 2003, pp. 17-30.

11. Ce sera aussi le cas quelques années plus tard avec la régence de Théophano, l'empire ottonien sera alors gouverné par une femme entre 984 et 991.

12. M. Welton, op. cit. note 5 .

13. A. von Euw, op. cit. note 3, pp. 37-48.

14. R. Le Jan, op. cit. note 6, p. 35.

15. Flodoard, Annales, a 951.

16. Berlin, Staatsbibliothek, MS theol. Lat. Fol. 58, fol. 120v.

17. La Haye, Royal Library, Hs. 76 F 1, fol. 214v.

18. T.O.B., 2010.

19. S. MacLean, Ottonian Queenship, Oxford, Oxford University Press, 2017, pp. 77-79.

20. Elizabeth Coatsworth, « Text and Textile », Studies Éamonn Ó Carragáin, s. 1., 2007, pp. 187-208.

21. A. Gajewski, op. cit. note 1.

22. V. L. Garver, op. cit. note 1.

23. Protévangile de Jacques, 11, 1-3, Pseudo-Matthieu 9, 1-2.

24. À titre de comparaison, Helen Stevens affirme que les broderies de Maaseik auraient nécessité huit mois de travail à plein temps. Helen M. Stevens, « Maaseik Reconstructed: A Practical Investigation and Interpretation of Eighth-Century Embroidery Techniques", Archaeological Textiles in Northern Europe, Copenhague, 1992, pp. 57-60.

25. A. Weyl Carr, op. cit. note 1.

26. Conservé à Bamberg, Diözesanmuseum, Inventar-Nr. 2728/3-5.

27. Charles Reginald Dodwell, Anglo-Saxon art: A new perspective, Manchester, 1982, vol. 3, p. 134.

28. Des incertitudes subsistent à propos de cette utilisation de la broderie de Bayeux, E. Coatsworth, op. cit. note 20, p. 196.

\section{RÉSUMÉS}

La bannière de la reine Gerberge est un outil de démonstration du pouvoir, fruit d'un contexte historique spécifique, reflétant à la fois la relation particulière des reines au pouvoir et à l'art de la broderie. Le medium de la broderie est un outil d'expression capital pour les femmes de l'élite durant le haut Moyen Âge, qui leur permet de laisser plus facilement leur trace en tant que commanditaires. L'image contourne ici l'impossibilité de représenter la femme guerrière, et offre, de par sa matérialité et son iconographie impériale triomphale, une démonstration de puissance. Elle est aussi symptomatique d'une époque à laquelle les souverains, en mal de 
légitimité dynastique vis-à-vis d'un pouvoir qu'ils ont usurpé, cherchent à consolider, ici par l'image, leur lignée dans un échiquier politique instable.

The standard of Queen Gerberge is a device to demonstrate power, the outcome of a specific historical context, and it reflects both the special relationship of queens with power and the art of embroidery. Embroidery was a key means of expression for the women of the elite class during the early Middle Ages, which allowed them to more easily leave their mark as forces of influence. The image here circumvents the impossibility of showing a woman as a warrior yet presents a demonstration of power through its materiality and its triumphant imperial iconography. It is also indicative of a time when sovereigns - in need of dynastic legitimacy for power they have usurped - attempted to consolidate their lineage, here with the use of imagery, on a volatile political chessboard.

\section{INDEX}

Index chronologique : haut moyen âge

Keywords : Ottonians, Carolingians, queen, gender, embroidery, textile, iconography, Early Middle Ages, representations, aristocracy

Mots-clés : ottoniens, carolingiens, reine, genre, broderie, textile, iconographie, représentations, aristocratie

\section{AUTEUR}

\section{MARJOLAINE MASSÉ}

Marjolaine Massé est doctorante au sein du CESCM de Poitiers, où elle prépare une thèse sous la direction de Cécile Voyer (Université de Poitiers) et d'Anne-Orange Poilpré (Université ParisPanthéon-Sorbonne), ayant pour titre «Les représentations des élites dans les images entre le IX ${ }^{\mathrm{e}}$ et le $\mathrm{XI}^{\mathrm{e}}$ siècle ». Elle est aussi chargée d'études et de recherche à l'INHA au sein du domaine Histoire de l'art du $\mathrm{IV}^{\mathrm{e}}$ au XV $\mathrm{XV}^{\mathrm{e}}$ siècle depuis octobre 2017.

Marjolaine Massé is a doctoral student at the CESCM in Poitiers, where she is preparing a dissertation under the direction of Cécile Voyer (Université de Poitiers) and Anne-Orange Poilpré (Université Paris-Panthéon-Sorbonne) titled "Representations of the elites in images from the 9th to 11th centuries". Since October 2017 she has also been a study and research fellow at the INHA in the history of art from the 4th to the 15th centuries. 